\title{
Modified Kantorovich-Stancu operators (II)
}

Ioan Gavrea and Adonia-Augustina Opriş

Abstract. In this paper, we introduce a new kind of Bernstein-KantorovichStancu operators. These operators generalize the operators introduced in the paper [2] by V. Gupta, G. Tachev and A.M. Acu.

Mathematics Subject Classification (2010): 41A25, 41A36.

Keywords: Approximation by linear operators, Kantorovich-Stancu operators.

\section{Introduction}

For $f \in C([0,1])$, the Bernstein operator of degree $n$ is defined by

$$
B_{n}(f ; x)=\sum_{k=0}^{n} p_{n, k}(x) f\left(\frac{k}{n}\right), x \in[0,1]
$$

where

$$
p_{n, k}(x)=\left(\begin{array}{l}
n \\
k
\end{array}\right) x^{k}(1-x)^{n-k}, k=0,1, \ldots, n
$$

and $p_{n, k}(x)=0$ if $k<0$ or $k>n$.

In [3], H. Khosravian-Arab, M. Delghan and M.R. Eslahchi, starting from well-known equalities

$$
p_{n, k}(x)=(1-x) p_{n-1, k}(x)+x p_{n-1, k-1}(x)
$$

and

$$
p_{n, k}(x)=(1-x)^{2} p_{n-2, k}(x)+2 x(1-x) p_{n-2, k-1}(x)+x^{2} p_{n-2, k-2}(x), 0<k<n
$$

have introduced modified Bernstein operators:

(i) $B_{n}^{M, 1}$ defined by

$$
B_{n}^{M, 1}(f ; x)=\sum_{k=0}^{n} p_{n, k}^{M, 1}(x) f\left(\frac{k}{n}\right), x \in[0,1]
$$

This paper has been presented at the fourth edition of the International Conference on Numerical Analysis and Approximation Theory (NAAT 2018), Cluj-Napoca, Romania, September 6-9, 2018. 
where

and

$$
p_{n, k}^{M, 1}=a(x, n) p_{n-1, k}(x)+a(1-x, n) p_{n-1, k-1}(x)
$$

(ii) $B_{n}^{M, 2}$ defined by

$$
a(x, n)=a_{1}(n) x+a_{0}(n), n=0,1, \ldots
$$

$$
B_{n}^{M, 2}(f ; x)=\sum_{k=0}^{n} p_{n, k}^{M, 2}(x) f\left(\frac{k}{n}\right), x \in[0,1]
$$

where

$$
p_{n, k}^{M, 2}(x)=b(x, n) p_{n-2, k}(x)+d(x, n) p_{n-2, k-1}(x)+b(1-x, n) p_{n-2, k-2}(x)
$$

and

$$
\begin{aligned}
& b(x, n)=b_{2}(n) x^{2}+b_{1}(n) x+b_{0}(n), \\
& d(x, n)=d_{0}(n) x(1-x), n=0,1, \ldots
\end{aligned}
$$

$a_{0}(n), a_{1}(n), b_{0}(n), b_{1}(n), b_{2}(n)$ and $d_{0}(n)$ are the unknown sequences which are determined in appropriate way for each forms.

V. Gupta, G. Tachev and A.M. Acu ([2]) have considered the operators:

$$
K_{n}^{M, 1}(f ; x)=(n+1) \sum_{k=0}^{n} p_{n, k}^{M, 1}(x) \int_{\frac{k}{n+1}}^{\frac{k+1}{n+1}} f(s) d s
$$

and

$$
K_{n}^{M, 2}(f ; x)=(n+1) \sum_{k=0}^{n} p_{n, k}^{M, 1}(x) \int_{\frac{k}{n+1}}^{\frac{k+1}{n+1}} f(s) d s .
$$

Here, they have discussed a uniform convergence estimate for these modified operators. In 1968, D.D. Stancu ([5]) has introduced the linear positive operators

$$
P_{n}^{(\alpha, \beta)}: C([0,1]) \rightarrow C([0,1])
$$

defined by

$$
P_{n}^{(\alpha, \beta)}(f ; x)=\sum_{k=0}^{n} p_{n, k}(x) f\left(\frac{k+\alpha}{n+\beta}\right)
$$

where $\alpha, \beta$ are two fixed real numbers such that $0 \leq \alpha \leq \beta$.

In 2004, D. Bărbosu ([1]) has introduced Kantorovich-Stancu operators

$$
K_{n}^{(\alpha, \beta)}: L_{1}([0,1]) \rightarrow C([0,1])
$$

defined by

$$
K_{n}^{(\alpha, \beta)}(f ; x)=(n+\beta+1) \sum_{k=0}^{n} p_{n, k}(x) \int_{\frac{k+\alpha}{n+\beta+1}}^{\frac{k+\alpha+1}{n+\beta+1}} f(s) d s .
$$

Regarding the previously modified operators, we note the following:

(a) The operators $B_{n}^{M, 1}$ and $B_{n}^{M, 2}$ are linear combinations of the operators $P_{n-1}^{(0,1)}$ and $P_{n-1}^{(1,1)}$, respectively of the operators $P_{n-2}^{(0,2)}, P_{n-2}^{(1,2)}$ and $P_{n-2}^{(2,2)}$, more precisely

$$
B_{n}^{M, 1}(f ; x)=a(x, n) P_{n-1}^{(0,1)}(f ; x)+a(1-x, n) P_{n-1}^{(1,1)}(f ; x)
$$


and

$$
B_{n}^{M, 2}(f ; x)=b(x, n) P_{n-2}^{(0,2)}(f ; x)+d(x, n) P_{n-2}^{(1,2)}(f ; x)+b(1-x, n) P_{n-2}^{(2,2)}(f ; x) ;
$$

(b) The operators $K_{n}^{M, 1}$ and $K_{n}^{M, 2}$ are linear combinations of the operators $K_{n-1}^{(0,1)}$ and $K_{n-1}^{(1,1)}$, respectively of the operators $K_{n-2}^{(0,2)}, K_{n-2}^{(1,2)}$ and $K_{n-2}^{(2,2)}$, therefore

$$
K_{n}^{M, 1}(f ; x)=a(x, n) K_{n-1}^{(0,1)}(f ; x)+a(1-x, n) K_{n-1}^{(1,1)}(f ; x)
$$

and

$$
K_{n}^{M, 2}(f ; x)=b(x, n) K_{n-2}^{(0,2)}(f ; x)+d(x, n) K_{n-2}^{(1,2)}(f ; x)+b(1-x, n) K_{n-2}^{(2,2)}(f ; x) .
$$

From the above reasons, in this paper we introduce, for any $\alpha, \beta \in \mathbb{R}, 0 \leq \alpha \leq \beta$ the operators

$$
\bar{K}_{n}^{(\alpha, \beta)}(f ; x)=(n+\beta+1) \sum_{k=0}^{n} p_{m, k}^{M, 1}(x) \int_{\frac{k+\alpha}{n+\beta+1}}^{\frac{k+\alpha+1}{n+\beta+1}} f(s) d s
$$

and

$$
\overline{\bar{K}}_{n}^{(\alpha, \beta)}(f ; x)=(n+\beta+1) \sum_{k=0}^{n} p_{m, k}^{M, 2}(x) \int_{\frac{k+\alpha}{n+\beta+1}}^{\frac{k+\alpha+1}{n+\beta+1}} f(s) d s .
$$

We mention that the Kantorovich-Stancu type operators $\bar{K}_{n}^{(\alpha, \beta)}$ was studied in a recent paper submitted for publication ([4]).

\section{Auxiliary results}

Lemma 2.1. The central moments of $K_{n}^{(\alpha, \beta)}$ are given by:

$$
\begin{gathered}
K_{n}^{(\alpha, \beta)}\left((t-x)^{s} ; x\right)=\frac{1}{s+1} \sum_{k=0}^{n} p_{n, k}(x)\left\{\sum_{i=0}^{s+1}\left(\begin{array}{c}
s+1 \\
i
\end{array}\right)\left(\frac{k+1}{n+1}-x\right)^{i} \frac{1}{(n+\beta+1)^{s-i}}\right. \\
\left.\times\left[\sum_{j=1}^{s+1-i}(-1)^{j+1}\left(\begin{array}{c}
s+1-i \\
j
\end{array}\right)\left(\alpha-\beta \frac{k+1}{n+1}\right)^{s+1-i-j}\right]\right\} .
\end{gathered}
$$

Proof.

$$
\begin{gathered}
K_{n}^{(\alpha, \beta)}\left((t-x)^{s} ; x\right)=(n+\beta+1) \sum_{k=0}^{n} p_{n, k}(x) \int_{\frac{k+\alpha}{n+\beta+1}}^{\frac{k+\alpha+1}{n+\beta+1}}(t-x)^{s} d t \\
=(n+\beta+1) \frac{1}{s+1} \sum_{k=0}^{n} p_{n, k}(x)\left[\left(\frac{k+\alpha+1}{n+\beta+1}-x\right)^{s+1}-\left(\frac{k+\alpha}{n+\beta+1}-x\right)^{s+1}\right]
\end{gathered}
$$

Because

$$
\begin{aligned}
\frac{k+\alpha+1}{n+\beta+1}-x & =\frac{k+1}{n+1}-x+\frac{k+\alpha+1}{n+\beta+1}-\frac{k+1}{n+1} \\
& =\frac{k+1}{n+1}-x+\left(\alpha-\beta \frac{k+1}{n+1}\right) \frac{1}{n+\beta+1},
\end{aligned}
$$


we have

$$
\begin{gathered}
\left(\frac{k+\alpha+1}{n+\beta+1}-x\right)^{s+1} \\
=\sum_{i=0}^{s+1}\left(\begin{array}{c}
s+1 \\
i
\end{array}\right)\left(\frac{k+1}{n+1}-x\right)^{i}\left(\alpha-\beta \frac{k+1}{n+1}\right)^{s+1-i} \frac{1}{(n+\beta+1)^{s+1-i}}
\end{gathered}
$$

and

$$
\begin{gathered}
\left(\frac{k+\alpha}{n+\beta+1}-x\right)^{s+1} \\
=\sum_{i=0}^{s+1}\left(\begin{array}{c}
s+1 \\
i
\end{array}\right)\left(\frac{k+1}{n+1}-x\right)^{i}\left(\alpha-1-\beta \frac{k+1}{n+1}\right)^{s+1-i} \frac{1}{(n+\beta+1)^{s+1-i}} .
\end{gathered}
$$

So, (2.2) becomes

$$
\begin{aligned}
K_{n}^{(\alpha, \beta)}\left((t-x)^{s}\right. & ; x)=\frac{1}{s+1} \sum_{k=0}^{n} p_{n, k}(x)\left\{\sum_{i=0}^{s+1}\left(\begin{array}{c}
s+1 \\
i
\end{array}\right)\left(\frac{k+1}{n+1}-x\right) \frac{1}{(n+\beta+1)^{s-i}}\right. \\
& \left.\times\left[\left(\alpha-\beta \frac{k+1}{n+1}\right)^{s+1-i}-\left(\alpha-1-\beta \frac{k+1}{n+1}\right)^{s+1-i}\right]\right\} \\
= & \frac{1}{s+1} \sum_{k=0}^{n} p_{n, k}(x)\left\{\sum_{i=0}^{s+1}\left(\begin{array}{c}
s+1 \\
i
\end{array}\right)\left(\frac{k+1}{n+1}-x\right)^{i} \frac{1}{(n+\beta+1)^{s-i}}\right. \\
& \left.\times\left[\sum_{j=1}^{s+1-i}(-1)^{j+1}\left(\begin{array}{c}
s+1-i \\
j
\end{array}\right)\left(\alpha-\beta \frac{k+1}{n+1}\right)^{s+1-i-j}\right]\right\} .
\end{aligned}
$$

Remark 2.2. For $s=\overline{1,6}$ we have

$$
\begin{gathered}
K_{n}^{(\alpha, \beta)}(t-x ; x)=-\frac{\beta+1}{n+\beta+1} x+\frac{2 \alpha+1}{2(n+\beta+1)}, \\
K_{n}^{(\alpha, \beta)}\left((t-x)^{2} ; x\right)=\frac{n-(2 \alpha+1)(\beta+1)}{(n+\beta+1)^{2}} x(1-x) \\
+\frac{(\beta-2 \alpha)(\beta+1)}{(n+\beta+1)^{2}} x^{2}+\frac{3 \alpha^{2}+3 \alpha+1}{3(n+\beta+1)^{2}} \\
+\frac{(12 \alpha+10) n-6(2 \alpha+1)(\beta+1)^{2}+4(\beta+1)^{3}}{4(n+\beta+1)^{3}} x(1-x) \\
K_{n}^{(\alpha, \beta)}\left((t-x)^{3} ; x\right)=-\frac{(3 \beta+5) n-(\beta+1)^{3}}{(n+\beta+1)^{3}} x^{2}(1-x) \\
-\frac{4\left(3 \alpha^{2}+3 \alpha+1\right)(\beta+1)-6(2 \alpha+1)(\beta+1)^{2}+4(\beta+1)^{3}}{4(n+\beta+1)^{3}} x \\
+\frac{4 \alpha^{3}+6 \alpha^{2}+4 \alpha+1}{4(n+\beta+1)^{3}}, \\
K_{n}^{(\alpha, \beta)}\left((t-x)^{4} ; x\right)=\frac{3 n^{2}-2\left(3+4(\beta+1)+3(\beta+1)^{2}\right) n}{(n+\beta+1)^{4}}(x(1-x))^{2}
\end{gathered}
$$




$$
\begin{aligned}
& -\frac{\left[4(2 \alpha+1)+2(6 \alpha+1)(\beta+1)-6(\beta+1)^{2}\right] n-2(2 \alpha+1)(\beta+1)^{3}}{(n+\beta+1)^{4}} x^{2}(1-x) \\
& +\frac{\left(6 \alpha^{2}+10 \alpha+5\right) n+2(2 \alpha+1)(\beta+1)^{3}-2\left(3 \alpha^{2}+3 \alpha+1\right)(\beta+1)^{2}}{(n+\beta+1)^{4}} x(1-x) \\
& -\frac{2(2 \alpha+1)(\beta+1)^{3}-2\left(3 \alpha^{2}+3 \alpha+1\right)(\beta+1)^{2}+\left(4 \alpha^{3}+6 \alpha^{2}+4 \alpha+1\right)(\beta+1)}{(n+\beta+1)^{4}} x \\
& +\frac{5 \alpha^{4}+10 \alpha^{3}+10 \alpha^{2}+5 \alpha+1}{5(n+\beta+1)^{4}}, \\
& K_{n}^{(\alpha, \beta)}\left((t-x)^{5} ; x\right)=\frac{\left[(30 \beta+70) x^{2}(1-x)^{3}+(30 \alpha-30 \beta-35) x^{2}(1-x)^{2}\right]}{2(n+\beta+1)^{5}} n^{2} \\
& +\left[-\frac{30(\beta+1)^{3}+60(\beta+1)^{2}+90(\beta+1)+72}{3(n+\beta+1)^{5}} x^{2}(1-x)^{3}\right. \\
& +\frac{60(\beta+1)^{3}-45(2 \alpha-1)(\beta+1)^{2}-30(4 \alpha-1)(\beta+1)-9(10 \alpha+1)}{3(n+\beta+1)^{5}} x^{2}(1-x)^{2} \\
& -\frac{30(\beta+1)^{3}-15(6 \alpha+1)(\beta+1)^{2}+15\left(6 \alpha^{2}+2 \alpha+1\right)(\beta+1)+2\left(30 \alpha^{2}+30 \alpha+13\right)}{3(n+\beta+1)^{5}} x^{2}(1-x) \\
& \left.+\frac{30 \alpha^{3}+75 \alpha^{2}+75 \alpha+28}{3(n+\beta+1)^{5}} x(1-x)\right] n+O\left(\frac{1}{n^{5}}\right) \\
& K_{n}^{(\alpha, \beta)}\left((t-x)^{6} ; x\right)=\frac{15 x^{3}(1-x)^{3}}{(n+\beta+1)^{6}} n^{3}+\left[\frac{45(\beta+1)^{2} x^{4}(1-x)^{2}}{(n+\beta+1)^{6}}\right. \\
& -\frac{\left(120 x^{3}(1-x)^{3}+15(6 \alpha-1) x^{3}(1-x)^{2}\right)(\beta+1)}{(n+\beta+1)^{6}} \\
& \left.+\frac{130 x^{2}(1-x)^{4}+10(12 \alpha-7) x^{2}(1-x)^{3}+5\left(9 \alpha^{2}-3 \alpha+2\right) x^{2}(1-x)^{2}}{(n+\beta+1)^{6}}\right] n^{2}+O\left(\frac{1}{n^{5}}\right) \text {. }
\end{aligned}
$$

\section{Main results} by (1.6).

Here, we will extend the results from [4] for modified operators $\overline{\bar{K}}_{n}^{(\alpha, \beta)}$ defined

It is easy to see that

$$
\begin{aligned}
\overline{\bar{K}}_{n}^{(\alpha, \beta)}(f ; x) & =b(x ; n) K_{n-2}^{(\alpha, \beta+2)}(f ; x)+d(x ; n) K_{n-2}^{(\alpha+1, \beta+2)}(f ; x) \\
& +b(1-x ; n) K_{n-2}^{(\alpha+2, \beta+2)}(f ; x) .
\end{aligned}
$$


Lemma 3.1. For $i=0,1,2$, the moments of $\overline{\bar{K}}_{n}\left(t^{i} ; x\right)$ are given by:

$$
\begin{aligned}
& \overline{\bar{K}}_{n}^{(\alpha, \beta)}(1 ; x)=\left(2 b_{2}(n)-d_{0}(n)\right) x^{2}-\left(2 b_{2}(n)-d_{0}(n)\right) x+b_{2}(n)+b_{1}(n)+2 b_{0}(n), \\
& \overline{\bar{K}}_{n}^{(\alpha, \beta)}(t ; x)=\frac{(2(n-2) x+2 \alpha+3)\left(2 b_{2}(n)-d_{0}(n)\right)}{2(n+\beta+1)} x(x-1) \\
& +\frac{(n-4)\left(b_{2}(n)+b_{1}(n)\right)+2(n-2) b_{0}(n)}{n+\beta+1} x \\
& +\frac{(2 \alpha+5)\left(b_{2}(n)+b_{1}(n)\right)+2(2 \alpha+3) b_{0}(n)}{2(n+\beta+1)}, \\
& \overline{\bar{K}}_{n}^{(\alpha, \beta)}\left(t^{2} ; x\right)=\left[\left(2 b_{2}(n)-d_{0}(n)\right) x(x-1)+b_{2}(n)+b_{1}(n)+2 b_{0}(n)\right] \\
& \times\left[\frac{(n-2)(n-3)}{(n+\beta+1)^{2}} x^{2}+\frac{2(\alpha+1)(n-2)}{(n+\beta+1)^{2}} x+\frac{3 \alpha^{2}+3 \alpha+1}{3(n+\beta+1)^{2}}\right] \\
& +\left[\frac{2\left(2 b_{2}(n)-d_{0}(n)\right) x^{3}}{(n+\beta+1)^{2}}-\frac{\left(2\left(2 b_{2}(n)-d_{0}(n)\right)+4\left(b_{2}(n)+b_{1}(n)\right)\right) x^{2}}{(n+\beta+1)^{2}}\right. \\
& \left.+\frac{4\left(b_{2}(n)+b_{1}(n)+b_{0}(n)\right)}{(n+\beta+1)^{2}} x\right](n-2) \\
& +\frac{2(\alpha+1)\left(2 b_{2}(n)-d_{0}(n)\right)+2 b_{2}(n)}{(n+\beta+1)^{2}} x^{2} \\
& -\left[\frac{2(\alpha+1)\left(2 b_{2}(n)-d_{0}(n)\right)}{(n+\beta+1)^{2}}+\frac{4(\alpha+2) b_{2}(n)+2(2 \alpha+3) b_{1}(n)}{(n+\beta+1)^{2}}\right] x \\
& +\frac{2(2 \alpha+3)\left(b_{2}(n)+b_{1}(n)+b_{0}(n)\right)}{(n+\beta+1)^{2}} .
\end{aligned}
$$

We want to demonstrate the uniform convergence of the sequence $\left(\overline{\bar{K}}_{n}^{(\alpha, \beta)} f\right)_{n \geq 2}$. For this purpose, we will consider that

$$
\begin{gathered}
\overline{\bar{K}}_{n}^{(\alpha, \beta)}(1 ; x)=1 \\
\Leftrightarrow 2 b_{2}(n)-d_{0}(n)=0 \text { and } b_{2}(n)+b_{1}(n)+2 b_{0}(n)=1 .
\end{gathered}
$$

Using these, we obtain that

$$
\begin{gathered}
\overline{\bar{K}}_{n}^{(\alpha, \beta)}(t ; x)=x+\frac{4 b_{0}(n)-\beta-5}{n+\beta+1} x+\frac{2 \alpha+5-4 b_{0}(n)}{2(n+\beta+1)} \\
\overline{\bar{K}}_{n}^{(\alpha, \beta)}\left(t^{2} ; x\right)=\frac{n^{2}-\left(9-8 b_{0}(n)\right) n+16-2 b_{1}(n)-20 b_{0}(n)}{(n+\beta+1)^{2}} x^{2} \\
+\frac{2\left(\alpha+3-2 b_{0}(n)\right) n+2 b_{1}(n)+8(\alpha+3) b_{0}(n)-4(2 \alpha+5)}{(n+\beta+1)^{2}} x \\
+\frac{3 \alpha^{2}+15 \alpha+19-6(2 \alpha+3) b_{0}(n)}{3(n+\beta+1)^{2}} .
\end{gathered}
$$

Assume that $\beta=2 \alpha$, for $b_{0}(n)=\frac{\beta+5}{4}$ the above expressions become

$$
\widetilde{K}_{n}^{(\alpha, \beta)}(t ; x)=x
$$




$$
\widetilde{K}_{n}^{(\alpha, \beta)}\left(t^{2} ; x\right)=x^{2}+\frac{n+2 b_{1}(n)+(\beta+2)(\beta+5)}{(n+\beta+1)^{2}} x(1-x)-\frac{3 \beta^{2}+18 \beta+14}{12(n+\beta+1)^{2}} .
$$

Taking $b_{1}(n)=-\frac{n+(\beta+2)(\beta+5)}{2}$ we have that

$$
\widetilde{K}_{n}^{(\alpha, \beta)}\left(t^{2} ; x\right)=x^{2}-\frac{3 \beta^{2}+18 \beta+14}{12(n+\beta+1)^{2}} \text {. }
$$

By (3.2) we obtain

and

$$
b_{2}(n)=\frac{n+2+(\beta+1)(\beta+5)}{2}
$$

$$
d_{0}(n)=n+2+(\beta+1)(\beta+5) .
$$

In this situation, we can give other expressions for the first six central moments.

\section{Lemma 3.2.}

$$
\begin{gathered}
\widetilde{K}_{n}^{(\alpha, \beta)}(t-x ; x)=0 \\
\widetilde{K}_{n}^{(\alpha, \beta)}\left((t-x)^{2} ; x\right)=-\frac{3 \beta^{2}+18 \beta+14}{12(n+\beta+1)^{2}}, \\
\widetilde{K}_{n}^{(\alpha, \beta)}\left((t-x)^{3} ; x\right)=-\frac{1-2 x}{4(n+\beta+1)^{3}}[2(3 \beta+7) n x(1-x) \\
\left.+2\left(\beta^{3}+9 \beta^{2}+21 \beta+13\right) x(1-x)+\beta^{3}+9 \beta^{2}+23 \beta+15\right], \\
\widetilde{K}_{n}^{(\alpha, \beta)}\left((t-x)^{4} ; x\right)=\frac{-3 n^{2}}{(n+\beta+1)^{4}} x^{2}(1-x)^{2}+O\left(\frac{1}{n^{3}}\right), \\
\widetilde{K}_{n}^{(\alpha, \beta)}\left((t-x)^{5} ; x\right)=\frac{15(\beta+3) n^{2}}{(n+\beta+1)^{5}} x^{2}(1-x)^{2}(2 x-1)+O\left(\frac{1}{n^{4}}\right), \\
\widetilde{K}_{n}^{(\alpha, \beta)}\left((t-x)^{6} ; x\right)=\frac{-30 n^{3}}{(n+\beta+1)^{6}} x^{3}(1-x)^{3}+O\left(\frac{1}{n^{4}}\right) .
\end{gathered}
$$

Using this, we will prove the following result:

Theorem 3.3. For $x \in[0,1]$, if $f \in C^{(6)}([0,1])$, we have

$$
\widetilde{K}_{n}^{(\alpha, \beta)}(f ; x)-f(x)=O\left(\frac{1}{n^{2}}\right),
$$

for sufficient large $n$.

Proof. Applying the Taylor's formula to the operators $\widetilde{K}_{n}^{(\alpha, \beta)}$ we have

$$
\begin{aligned}
\widetilde{K}_{n}^{(\alpha, \beta)}(f ; x) & =f(x)+\sum_{k=1}^{6} \frac{1}{k !} \widetilde{K}_{n}^{(\alpha, \beta)}\left((t-x)^{k} ; x\right) f^{(k)}(x) \\
& +\widetilde{K}_{n}^{(\alpha, \beta)}\left(\rho(t ; x)(t-x)^{6} ; x\right),
\end{aligned}
$$

where $\rho$ is a continuous function.

It is sufficient to prove that

$$
\left|\widetilde{K}_{n}^{(\alpha, \beta)}\left(\rho(t ; x)(t-x)^{6} ; x\right)\right|=O\left(\frac{1}{n^{2}}\right) .
$$


We know that operators are not positive, so we rewrite them like this

$$
\widetilde{K}_{n}^{(\alpha, \beta)}(f ; x)=\widetilde{K}_{n, 1}^{(\alpha, \beta)}(f ; x)-\widetilde{K}_{n, 2}^{(\alpha, \beta)}(f ; x)
$$

where

$$
\begin{aligned}
\widetilde{K}_{n, 1}^{(\alpha, \beta)}(f ; x)=\left(b_{2}(n) x^{2}+b_{0}(n)\right) \cdot K_{n-2}^{(\alpha, \beta+2)}(f ; x) & +d_{0}(n) x \cdot K_{n-2}^{(\alpha+1, \beta+2)}(f ; x) \\
& +b_{2}(n) x^{2} \cdot K_{n-2}^{(\alpha+2, \beta+2)}(f ; x)
\end{aligned}
$$

and

$$
\begin{aligned}
& \widetilde{K}_{n, 2}^{(\alpha, \beta)}(f ; x)=-b_{1}(n) x \cdot K_{n-2}^{(\alpha, \beta+2)}(f ; x)+d_{0}(n) x^{2} \cdot K_{n-2}^{(\alpha+1, \beta+2)}(f ; x) \\
& \quad+\left(\left(2 b_{2}(n)+b_{1}(n)\right) x-\left(b_{2}(n)+b_{1}(n)+b_{0}(n)\right)\right) \cdot K_{n-2}^{(\alpha+2, \beta+2)}(f ; x) .
\end{aligned}
$$

We note that $\widetilde{K}_{n, 1}^{(\alpha, \beta)}$ and $\widetilde{K}_{n, 2}^{(\alpha, \beta)}$ are linear and positive operators.

$$
\begin{aligned}
\left|\widetilde{K}_{n}^{(\alpha, \beta)}\left(\rho(t ; x)(t-x)^{6} ; x\right)\right| & \leq\left|\widetilde{K}_{n, 1}^{(\alpha, \beta)}\left(\rho(t ; x)(t-x)^{6} ; x\right)\right| \\
& +\left|\widetilde{K}_{n, 2}^{(\alpha, \beta)}\left(\rho(t ; x)(t-x)^{6} ; x\right)\right| .
\end{aligned}
$$

Computing $\widetilde{K}_{n, i}^{(\alpha, \beta)}\left((t-x)^{6} ; x\right), i=1,2$, we obtain the following expressions

$$
\widetilde{K}_{n, i}^{(\alpha, \beta)}\left((t-x)^{6} ; x\right)=\frac{15 x^{4}(1-x)^{3}(1+x) n^{4}}{(n+\beta+1)^{6}}+A_{i}(\alpha, \beta, x) \frac{n^{3}}{(n+\beta+1)^{6}}, i=1,2
$$

where

$$
\begin{gathered}
A_{1}(\alpha, \beta, x)=15\left(\beta^{2}+6 \beta+7\right) x^{4}(1-x)^{3}(1+x) \\
-\left[120 x^{4}(1-x)^{3}(1+x)+15(6 \alpha+5) x^{4}(1-x)^{2}(1+x)\right](\beta+3) \\
+45 x^{5}(1-x)^{2}(1+x)(\beta+3)^{2}+130 x^{3}(1-x)^{4}(1+x) \\
+10(12 \alpha+5) x^{3}(1-x)^{3}(1+x)+5\left(9 \alpha^{2}+15 \alpha+8\right) x^{3}(1-x)^{2}(1+x) \\
+45 x^{4}(1-x)^{2}+\frac{15(\beta+5) x^{3}(1-x)^{3}}{4}
\end{gathered}
$$

and

$$
\begin{gathered}
A_{2}(\alpha, \beta, x)=15\left(\beta^{2}+6 \beta+7\right) x^{4}(1-x)^{3}(1+x) \\
-\left[120 x^{4}(1-x)^{3}(1+x)+15(6 \alpha+5) x^{4}(1-x)^{2}(1+x)\right](\beta+3) \\
+45 x^{5}(1-x)^{2}(1+x)(\beta+3)^{2}+130 x^{3}(1-x)^{4}(1+x) \\
+10(12 \alpha+5) x^{3}(1-x)^{3}(1+x)+5\left(9 \alpha^{2}+15 \alpha+8\right) x^{3}(1-x)^{2}(1+x) \\
+45 x^{3}(1-x)^{2}-\frac{15(\beta+1) x^{3}(1-x)^{3}}{4} .
\end{gathered}
$$

Because $\rho$ is a continuous function, there exists an $M>0$ such that $|\rho(t ; x)|<M$, $\forall x, t \in[0,1]$. Using the above results for $\widetilde{K}_{n, i}^{(\alpha, \beta)}\left((t-x)^{6} ; x\right)$, we obtain

$$
\begin{aligned}
\left|\widetilde{K}_{n, i}^{(\alpha, \beta)}\left(\rho(t ; x)(t-x)^{6} ; x\right)\right| & \leq M\left|\frac{15 x^{4}(1-x)^{3}(1+x) n^{4}}{(n+\beta+1)^{6}}+O\left(\frac{1}{n^{3}}\right)\right| \\
& =O\left(\frac{1}{n^{2}}\right), i=1,2 .
\end{aligned}
$$

So, (3.4) is proved.

Combining this with Lemma 3.2, we complete the proof of theorem. 


\title{
References
}

[1] Bărbosu, D., Kantorovich-Stancu type operators, Journal of Inequalities in Pure and Applied Mathematics, 5(2004), no. 3, Article 53.

[2] Gupta, V., Tachev, G., Acu, A.M., Modified Kantorovich operators with better approximation properties, Numer. Algo. (2018). https://doi.org/10.1007/s11075-018-0538-7

[3] Khosravian-Arab, H., Dehghan, M., Eslahchi, M.R., A new approach to improve the order of approximation of the Bernstein operators: theory and applications, Numer. Algo., 77(2018), no. 1, 111-150.

[4] Opriş, A.A., Approximation by modified Kantorovich-Stancu operators, JIAP-D-1800423, april, 2018 (summited for publication).

[5] Stancu, D.D., Asupra unei generalizări a polinoamelor lui Bernstein, Studia Univ. Babeş-Bolyai, Ser. Math.-Phys., 14(1969), no. 2, 31-45.

\author{
Ioan Gavrea \\ Department of Mathematics \\ Technical University of Cluj-Napoca \\ str. Memorandumului, nr. 28, 400144 \\ Cluj-Napoca, Romania \\ e-mail: ioan.gavrea@math.utcluj.ro \\ Adonia-Augustina Opriş \\ Department of Mathematics \\ Technical University of Cluj-Napoca \\ str. Memorandumului, nr. 28, 400144 \\ Cluj-Napoca, Romania \\ e-mail: mate.salaj@yahoo.com
}

